\title{
The New Useful High-Resolution Computed Tomography Finding for Diagnosing Fibrotic Hypersensitivity Pneumonitis: "Hexagonal Pattern": A Single-Center Retrospective Study
}

Hiroko Okabayashi ( $\nabla$ hirokokaba@hotmail.co.jp )

Kanagawa Cardiovascular and Respiratory Center

\section{Taiki Fukuda}

The Jikei University School of Medicine

\section{Tae Iwasawa}

Kanagawa Cardiovascular and Respiratory Center

Tsuneyuki Oda

Kanagawa Cardiovascular and Respiratory Center

Hideya Kitamura

Kanagawa Cardiovascular and Respiratory Center

\section{Tomohisa Baba}

Kanagawa Cardiovascular and Respiratory Center

\section{Tamiko Takemura}

Kanagawa Cardiovascular and Respiratory Center

\section{Takuro Sakagami}

Kumamoto University Hospital, Kumamoto University

\section{Takashi Ogura}

Kanagawa Cardiovascular and Respiratory Center

\section{Research Article}

Keywords: fibrotic hypersensitivity pneumonitis, idiopathic pulmonary pneumonitis, $\mathrm{HRCT}$, interlobular septal thickening, hexagonal pattern

Posted Date: November 17th, 2021

DOI: https://doi.org/10.21203/rs.3.rs-1077089/v1

License: (c) (1) This work is licensed under a Creative Commons Attribution 4.0 International License.

Read Full License 
Version of Record: A version of this preprint was published at BMC Pulmonary Medicine on March 4th, 2022. See the published version at https://doi.org/10.1186/s12890-022-01869-4. 


\section{Abstract \\ Background}

Centrilobular nodules, ground-glass opacity (GGO), mosaic attenuation, air trapping, and three-density pattern were reported as high-resolution computed tomography (HRCT) findings characteristic of fibrotic hypersensitivity pneumonitis (HP). However, it is often difficult to differentiate fibrotic HP from idiopathic pulmonary fibrosis (IPF). In fibrotic HP, the HRCT sometimes shows tortoiseshell-like interlobular septal thickening that extends from the subpleural lesion to the inner layers. This finding is called "hexagonal pattern," and this study is focused on the possibility that such finding is useful for differentiating fibrotic HP from IPF.

\section{Methods}

This study included patients with multidisciplinary discussion (MDD) diagnosis of fibrotic HP or IPF undergoing surgical lung biopsy between January 2015 and December 2017 in Kanagawa Cardiovascular and Respiratory Center. Two radiologists have evaluated the HRCT findings without clinical and pathological information.

\section{Results}

A total of 23 patients were diagnosed with fibrotic HP by MDD and 48 with IPF. Extensive GGO, centrilobular nodules, and hexagonal pattern were more frequent findings in fibrotic HP than in IPF. No significant difference was observed between the two groups in the presence or absence of mosaic attenuation, air trapping, or three-density pattern. In the multivariate logistic regression, the presence of extensive GGO and hexagonal pattern was associated with increased odds ratio of fibrotic HP. The sensitivity and specificity of the diagnosis of fibrotic HP in the presence of the hexagonal pattern were $69.6 \%$ and $87.5 \%$, respectively.

\section{Conclusion}

Hexagonal pattern is a useful finding for differentiating fibrotic HP from IPF.

\section{Background}

Two guidelines have recently been proposed for diagnosing hypersensitivity pneumonitis (HP), which state that lung biopsy is not necessary for the diagnosis of HP if antigen exposure, typical high-resolution computed tomography (HRCT) findings, and bronchoalveolar lavage (BAL) lymphocytosis have been identified [1, 2]. However, in fibrotic HP, the antigen is often unknown, and most cases need lung biopsy. In addition, specimens collected via transbronchial forceps biopsies or transbronchial lung cryobiopsy 
(TBLC) are insufficient in size for the diagnosis of fibrotic HP, and surgical lung biopsy (SLB) is often required. Conversely, SLB is often not feasible due to the patient's background or facility issues. Therefore, HRCT imaging plays a significant role in the diagnosis of fibrotic HP.

HRCT findings characteristic of fibrotic HP include centrilobular nodules, ground-glass opacity (GGO), mosaic attenuation, air trapping, and three-density pattern in previous reports [1-8]. These findings are not specific to fibrotic HP only, and many cases of fibrotic HP were found without typical HRCT findings.

Approximately half of chronic HP has been reported to have histological usual interstitial pneumonia (UIP)-like pattern and extensive fibrosis with poor prognosis [9]. Fibrotic HP with UIP pattern is common and often difficult to distinguish from idiopathic pulmonary fibrosis (IPF) in clinical practice. However, differentiating between fibrotic HP and IPF is important as they differ in their treatment strategies.

In fibrotic HP, the HRCT sometimes shows tortoiseshell-like interlobular septal thickening that extends from the subpleural region to the inner layers. This finding is called "hexagonal pattern," and this study focused on the possibility that such finding is useful for differentiating fibrotic HP from IPF (Figure 1).

\section{Methods}

\section{Patients}

This study included patients with multidisciplinary discussion (MDD) diagnosis of fibrotic HP or IPF undergoing SLB between January 2015 and December 2017 in Kanagawa Cardiovascular and Respiratory Center. The diagnosis of IPF and fibrotic HP was based on consensus using previously reported criteria [5,10-12]. The exclusion criteria of this study were as follows: (1) patients that had collagen vascular diseases or pulmonary alveolar proteinosis, (2) patients that met the interstitial pneumonia with autoimmune features diagnostic criteria [13], (3) patients that developed collagen vascular disease following MDD, and (4) patients that have already been given medication, including steroids or immunosuppressant, before biopsy.

The patient's baseline characteristics, including age, gender, smoking status, peripheral blood counts, blood biochemistry, pulmonary function tests, and BAL findings at the time of MDD, were retrospectively obtained from the medical records. This study was approved by the Institutional Review Board of Kanagawa Cardiovascular and Respiratory Center (permission number: KCRC-20-0023, permission date: August 18, 2020).

\section{The definition of hexagonal pattern}

Hexagonal pattern is defined as an interlobular septal thickening expanding from the subpleural region to two or more inner layers of secondary pulmonary lobules (Figure 1a, b). When there is subpleural collapse such that the structure of secondary pulmonary lobules is no longer recognizable, hexagonal pattern was defined as interlobular septal thickening that expands to two or more inner layers of secondary pulmonary lobules from the collapsed area (Figure 1c). 
Figure 2 presents the pathological specimen obtained via SLB of a patient with hexagonal pattern. Perilobular fibrosis and bridging fibrosis connecting the fibrosis in the centrilobular area and the fibrosis in the perilobular area were observed in this specimen. In this case, the hexagonal pattern observed in the HRCT corresponded to that of perilobular fibrosis.

\section{Radiological evaluation}

The radiological data were reviewed by two chest radiologists (T.I. and D.F.). HRCT images were independently evaluated, and the final findings were agreed upon by consensus between the two radiologists. The radiologists knew that only patients with fibrotic HP or IPF were included in the study, but they evaluated the HRCT findings without clinical and pathological information. The two radiologists have retrospectively evaluated the shadow distribution and the following findings: hexagonal pattern, extensive GGO, centrilobular nodules, mosaic attenuation, air trapping, three-density pattern, reticulation, traction bronchiectasis, honeycombing, consolidation, emphysema, cyst, and pleuroparenchymal fibroelastosis. The HRCT findings were interpreted based on the recommendations of the Nomenclature Committee of the Fleischner Society [14]. In this study, mosaic attenuation, air trapping, and three-density pattern were defined as being present when they were found in three or more lobules bilaterally. Before the radiological evaluation, two teacher images were shown to confirm the definition of hexagonal pattern.

\section{Statistical analysis}

Continuous variables are expressed as median values. The Mann-Whitney $U$ test, chi-squared test, or Fisher's exact probability test was employed, as appropriate, for between-group comparisons. The interobserver variation between two radiologists for the diagnosis of fibrotic HP or IPF was analyzed using $\mathrm{k}$ statistic. А к coefficient equal to or less than 0.20 indicated poor agreement; $0.21-0.40$, fair agreement; $0.41-0.60$, moderate agreement; $0.61-0.80$, good agreement; and $0.81-1.00$, excellent agreement. Univariate and multivariate logistic analyses were employed to identify the useful HRCT findings for distinguishing fibrotic HP from IPF. A $p$-value of $<0.05$ was considered to be significant. All statistical analyses have been conducted using the Statistical Package for the Social Sciences software version 24.0 (IBM; Armonk, NY, USA).

\section{Results}

\section{Patient characteristics}

During the study period, 216 patients diagnosed with interstitial lung disease underwent SLB at our hospital. A total of 28 patients were diagnosed with fibrotic HP by MDD at the time of SLB and 51 with IPF. Among the fibrotic HP patients, one had pulmonary alveolar proteinosis, and one had Sjögren's syndrome. Among the IPF patients, one met the diagnosis criteria of interstitial pneumonia with autoimmune features. One patient each with IPF and fibrotic HP received steroids prior to SLB. One patient with IPF developed rheumatoid arthritis after biopsy. Excluding cases that met the exclusion 
criteria, 23 cases of fibrotic HP and 48 cases of IPF were considered in this study (Figure 3 ). The American Thoracic Society (ATS)/Japanese Respiratory Society (JRS)/Latin American Thoracic Association (ALAT) Clinical Practice Guideline of the diagnosis of HP was published in 2020 [1], but the diagnosis in this study was made before this guideline was reported. The fibrotic HP diagnosis was again reviewed according to this guideline, and all cases of fibrotic HP diagnosed by MDD were found to meet the criteria for definite or high-confidence HP.

Table 1 presents the characteristic of the patients with fibrotic HP and IPF at the MDD. The serum Krebs von den Lungen- 6 and surfactant protein-D levels were higher in fibrotic HP than in IPF patients. The percent predicted forced vital capacity $(F V C)$, percent predicted forced expiratory volume in one second $\left(\mathrm{FEV}_{1}\right), \mathrm{FEV}_{1} / \mathrm{FVC}$ ratio, and percent predicted diffusion lung capacity for carbon monoxide $\left(\mathrm{DL}_{\mathrm{CO}}\right)$ at diagnosis were not significantly different between the two groups. The lymphocyte ratio and CD4/8 ratio in BAL were significantly higher among fibrotic HP patients. 
Table 1

Patient characteristics

\begin{tabular}{|c|c|c|c|}
\hline & Fibrotic HP n = 23 & IPF $n=48$ & $P$-value \\
\hline Age, years & $68(56-76)$ & $67(49-77)$ & 0.362 \\
\hline Gender (male/female), $\mathrm{n}$ & $15 / 8$ & $35 / 13$ & 0.506 \\
\hline Smoking status (ex/never) & $12 / 11$ & $32 / 16$ & 0.239 \\
\hline WBC, $\mu \mathrm{L}$ & $6250(2530-10300)$ & $6365(3400-13500)$ & 0.531 \\
\hline LDH, IU/L & $232(149-305)$ & $211(139-360)$ & 0.428 \\
\hline $\mathrm{CRP}, \mathrm{mg} / \mathrm{dL}$ & $0.11(0.01-1.50)$ & $0.14(0.01-1.79)$ & 0.526 \\
\hline $\mathrm{KL}-6, \mathrm{U} / \mathrm{mL}$ & $1772(578-8437)$ & $834(286-4666)$ & $<0.001$ \\
\hline SP-D, pg/mL & $393(84-1111)$ & $222(45-897)$ & 0.049 \\
\hline \multicolumn{4}{|l|}{ Pulmonary function } \\
\hline FVC \% pred & $87.1(63.8-125.5)$ & $85.8(44.9-137.1)$ & 0.461 \\
\hline $\mathrm{FEV}_{1} / \mathrm{FVC}$ ratio & $78.0(65.4-96.6)$ & $78.3(59.5-100.0)$ & 0.606 \\
\hline $\mathrm{FEV}_{1} \%$ pred & $87.9(67.0-115.4)$ & $83.5(52.1-129.9)$ & 0.363 \\
\hline $\mathrm{DL}_{\mathrm{CO}} \%$ pred & $78.5(49.9-125.1)$ & $76.5(29.1-123.5)$ & 0.588 \\
\hline \multicolumn{4}{|l|}{ BAL fluid ${ }^{a}$} \\
\hline Total cell count, $\times 10^{4} / \mu \mathrm{L}$ & $26.9(2.6-69.1)$ & $24.3(0.2-62.1)$ & 0.750 \\
\hline Macrophages, \% & $40.5(4.0-91.0)$ & $83.0(17.0-96.0)$ & $<0.001$ \\
\hline Lymphocytes, \% & $55.2(7.5-95.0)$ & $11.3(1.6-70.0)$ & $<0.001$ \\
\hline Neutrophils, \% & $1.4(0-10.0)$ & $2.0(0-39.0)$ & 0.425 \\
\hline Eosinophils, \% & $0.8(0-6.0)$ & $2.0(0-18.0)$ & 0.060 \\
\hline $\mathrm{CD} 4 / 8$ ratio & $3.9(0.2-9.8)$ & $1.7(0-8.4)$ & 0.005 \\
\hline \multicolumn{4}{|c|}{ Data are expressed as median (range) or numbers of patients. } \\
\hline \multicolumn{4}{|c|}{${ }^{a}$ for BALF, $n=20$ (fibrotic HP), $n=39$ (IPF) } \\
\hline \multicolumn{4}{|c|}{$\begin{array}{l}\text { HP, hypersensitivity pneumonitis; IPF, idiopathic pulmonary fibrosis; WBC, white blood cell; } L D H \text {, } \\
\text { lactate dehydrogenase; CRP, C-reactive protein; KL-6, Krebs von den Lungen-6; SP-D, surfactant } \\
\text { protein-D; FVC, forced vital capacity; FEV }{ }_{1} \text {, forced expiratory volume in one second; } \mathrm{DL}_{\mathrm{CO}} \text {, diffusion } \\
\text { lung capacity for carbon monoxide; BAL, bronchoalveolar lavage }\end{array}$} \\
\hline
\end{tabular}




\section{Hrct Assessment}

Interobserver agreement across the two radiologists in the HRCT findings other than pleuroparenchymal fibroelastosis was fair to good. The kappa coefficient of hexagonal pattern was 0.44 , indicating moderate agreement (Table 2). Extensive GGO, centrilobular nodules, and hexagonal pattern were more frequently observed in fibrotic HP than in IPF. A significant difference in axial distribution was also found between fibrotic HP and IPF. 
Table 2

HRCT findings

\begin{tabular}{|c|c|c|c|c|c|}
\hline & $\begin{array}{l}\text { Fibrotic HP n = } \\
23\end{array}$ & IPF & $\begin{array}{l}P \\
\text { value }\end{array}$ & $\begin{array}{l}\text { Intero } \\
\text { agree }\end{array}$ & erver \\
\hline & & 48 & & K & $95 \% \mathrm{Cl}$ \\
\hline Distribution (zonal), $\mathrm{n}$ & & & 0.611 & 0.22 & -0.49 \\
\hline Upper/middle predominant & 0 & 1 & & & \\
\hline Lower predominant & 19 & 43 & & & \\
\hline Diffuse & 4 & 4 & & & \\
\hline Distribution (axial), n & & & 0.001 & 0.36 & $0.16-0.55$ \\
\hline Peribronchovascular & 2 & 1 & & & \\
\hline Peripheral & 12 & 44 & & & \\
\hline $\begin{array}{l}\text { Peripheral with subpleural } \\
\text { sparing }\end{array}$ & 4 & 2 & & & \\
\hline Diffuse & 5 & 1 & & & \\
\hline Extensive GGO, $\mathrm{n}$ & 19 & 19 & 0.001 & 0.38 & $0.16-0.59$ \\
\hline Centrilobular nodules, $\mathrm{n}$ & 20 & 27 & 0.010 & 0.27 & $0.07-0.47$ \\
\hline Mosaic attenuation, $\mathrm{n}$ & 5 & 3 & 0.102 & 0.21 & $-0.12-0.55$ \\
\hline Air trapping, $\mathrm{n}^{\mathrm{a}}$ & 15 & 25 & 0.623 & 0.46 & $0.26-0.67$ \\
\hline Three-density pattern, $\mathrm{n}$ & 0 & 0 & - & & \\
\hline Reticulation, $\mathrm{n}$ & 23 & 48 & - & & \\
\hline Honeycombing, $n$ & 3 & 14 & 0.136 & 0.52 & $0.24-0.79$ \\
\hline Traction bronchiectasis, $\mathrm{n}$ & 23 & 48 & - & & \\
\hline Consolidation, $\mathrm{n}$ & 1 & 7 & 0.261 & 0.48 & $0.11-0.85$ \\
\hline Emphysema, $n$ & 7 & 21 & 0.283 & 0.59 & $0.39-0.79$ \\
\hline PPFE, n & 1 & 10 & 0.090 & 0.14 & $-0.35-0.64$ \\
\hline Cyst, n & 8 & 27 & 0.090 & 0.69 & $0.52-0.86$ \\
\hline
\end{tabular}

Data are expressed as numbers of patients.

a. One patient of IPF had no expiratory CT available.

HP, hypersensitivity pneumonitis; IPF, idiopathic pulmonary fibrosis; GGO, ground-glass opacity; PPFE, pleuroparenchymal fibroelastosis 


\begin{tabular}{|c|c|c|c|c|c|}
\hline & \multirow[t]{2}{*}{$\begin{array}{l}\text { Fibrotic HP } n= \\
23\end{array}$} & \multirow{2}{*}{$\begin{array}{l}\text { IPF } \\
n= \\
48\end{array}$} & \multirow[t]{2}{*}{$\begin{array}{l}P \\
\text { value }\end{array}$} & \multicolumn{2}{|c|}{$\begin{array}{l}\text { Interobserver } \\
\text { agreement }\end{array}$} \\
\hline & & & & $\mathbf{K}$ & $95 \% \mathrm{Cl}$ \\
\hline Hexagonal pattern, $n$ & 16 & 6 & $<0.001$ & 0.44 & $0.19-0.69$ \\
\hline \multicolumn{6}{|c|}{ Data are expressed as numbers of patients. } \\
\hline \multicolumn{6}{|c|}{ a. One patient of IPF had no expiratory CT available. } \\
\hline \multicolumn{6}{|c|}{$\begin{array}{l}\text { HP, hypersensitivity pneumonitis; IPF, idiopathic pulmonary fibrosis; GGO, ground-glass opacity; PPFE, } \\
\text { pleuroparenchymal fibroelastosis }\end{array}$} \\
\hline
\end{tabular}

Mosaic attenuation, air trapping, or three-density pattern were reported as findings suggestive of fibrotic HP $[1,2,6,7]$, but no significant difference was observed in this study (Table 2). The logistic regression for predicting fibrotic HP is presented in Table 3. In the univariate logistic regression, the presence of extensive GGO, centrilobular nodules, and hexagonal pattern was associated with increased odds ratio of fibrotic HP. Extensive GGO and hexagonal pattern were the remaining variables in multivariate logistic regression. The presence of hexagonal pattern was highly specific (87.5\%) for diagnosing fibrotic HP. The sensitivity for fibrotic HP diagnosis when hexagonal pattern was present was $69.6 \%$. The positive predictive value of hexagonal pattern was $72.7 \%$ for fibrotic HP diagnosis. 
Table 3

Logistic regression predicting fibrotic HP

\begin{tabular}{|c|c|c|c|}
\hline & Odds ratio & $95 \% \mathrm{Cl}$ & $P$-value \\
\hline \multicolumn{4}{|l|}{ Univariate logistic regression } \\
\hline \multicolumn{4}{|l|}{ Distribution (zonal) } \\
\hline Upper/middle & 0.00 & $0.00-\operatorname{lnf}$ & 0.991 \\
\hline Lower & 0.44 & $0.10-1.96$ & 0.282 \\
\hline Diffuse & reference & & \\
\hline \multicolumn{4}{|l|}{ Distribution (axial) } \\
\hline Peripheral & 0.05 & $0.01-0.51$ & 0.011 \\
\hline Peripheral with subpleural sparing & 0.40 & $0.03-6.18$ & 0.512 \\
\hline Peribronchovascular & 0.40 & $0.02-10.00$ & 0.577 \\
\hline Diffuse & Reference & & \\
\hline Extensive GGO & 7.25 & $2.13-24.65$ & 0.002 \\
\hline Centrilobular nodules & 5.19 & $1.36-19.82$ & 0.016 \\
\hline Mosaic attenuation & 4.17 & $0.90-19.28$ & 0.068 \\
\hline Air trapping & 1.65 & $0.59-4.63$ & 0.341 \\
\hline Honeycombing & 0.36 & $0.09-1.43$ & 0.147 \\
\hline Consolidation & 0.27 & $0.03-2.31$ & 0.229 \\
\hline Emphysema & 0.56 & $0.20-1.62$ & 0.285 \\
\hline PPFE & 0.17 & $0.02-1.44$ & 0.105 \\
\hline Cyst & 0.42 & $0.15-1.16$ & 0.094 \\
\hline Hexagonal pattern & 16.00 & $4.66-54.91$ & $<0.001$ \\
\hline \multicolumn{4}{|l|}{ Multivariate logistic regression } \\
\hline Extensive GGO & 4.86 & $1.20-19.70$ & 0.027 \\
\hline Centrilobular nodules & 1.45 & $0.30-7.08$ & 0.647 \\
\hline Hexagonal pattern & 11.00 & $2.70-44.70$ & 0.001 \\
\hline
\end{tabular}

Discussion 
This study has demonstrated that hexagonal pattern can be a useful finding for differentiating fibrotic HP from IPF.

HP is a diffuse interstitial pneumonia caused by immune response to inhaled antigen. Inhaled substances are usually most likely deposited at the level of the respiratory bronchioles, and small particles of $2.5 \mu \mathrm{m}$ or less reach the alveolar region via diffusion, are phagocytosed by alveolar macrophages, and then enter the lymphatics. The distribution of lymphatic vessels in the lungs is largely divided into two routes. One lymphatic flow follows the bronchovascular bundles to the hilum in the inner layers. The other route starts at the perivenular area in the secondary lobule, runs through the interlobular septa or subpleural lymphatic vessels, and ends at the hilum [15]. In experiments using inhaled antigens, the most frequent site of granuloma formation in response to antigens is from the respiratory bronchioles to the alveolar dusts, as has been reported [16]. The deposition of inhaled substances into the respiratory bronchial habit is thought to be involved in the formation of lobular central lesions, whereas deposition by lymphatic flow is thought to be involved in the fibrosis of the lobular margins, i.e., subpleural and interlobular septal predominance.

Centrilobular nodules, extensive GGO, mosaic attenuation, air trapping, diffuse axial distribution, and upper or mid-lung predominance have been reported as useful HRCT findings for fibrotic HP diagnosis in previous reports $[4,6,8,17]$. However, these studies differed in patient backgrounds or diagnostic methods, including with or without lung biopsy: some studies included nonfibrotic HP and fibrotic HP and other studies included other ILDs and IPF as control diseases.

The study comparing IPF with bird-related chronic HP that had histological UIP pattern reported that upper or mid-lung predominance and profuse micronodules were reported as key findings in chronic HP diagnosis [8]. The usefulness of the three-density pattern for differentiating fibrotic HP from IPF was reported, but in this study, not all patients underwent SLB [7]. The present study did not include cases with three-density pattern, and no significant difference in the presence of mosaic attenuation was observed between the two groups. This may be because this study included only those cases that required SLB as clinical information and radiological findings did not lead to the diagnosis. This suggests that the hexagonal pattern may be useful for differentiating fibrotic HP from IPF, even without a three-density pattern.

Fibrosis of HP manifesting as irregular reticulation is often visible on HRCT, which appears as thickened interlobular septa $[1,18,19]$. This finding usually correlates with the presence of fibrosis predominantly affecting the periphery of acini and the secondary lobule rather than the septa themselves. The study about HP in North India has reported that septal thickening was observed in nearly $30 \%$ of patients [20]. However, patients with IPF or other ILD with UIP pattern also often have irregular interlobular septal thickening [18].

This study proposed that not only the presence of interlobular septal thickening just below the pleura but also the extension of interlobular septal thickening to the inner layers, exhibiting a tortoiseshell-like pattern (hexagonal pattern), may be more characteristics of fibrotic HP than IPF. 
In the case presented in Figure 2, the area showing a hexagonal pattern was biopsied, and the hexagonal pattern in the HRCT was thought to correspond to perilobular fibrosis. However, in this study, SLB was not performed at selected sites with hexagonal pattern; thus, the HRCT findings and pathology in all patients enrolled have not been compared in this study. Since hexagonal pattern is a shadow that extends to the inner layers, in some cases, the shadow is found in the inner layers beyond the area that can be sampled by SLB.

Recently, the utility of TBLC has been reported in the diagnosis of diffuse lung disease [21-24]. Cryoprobe-retrieved specimens are larger than those of transbronchial forceps biopsies and less crush. TBLC tends to sample more proximal portion of the lung apart from the pleura compared with SLB. In the future, TBLC and SLB may be useful for comparing the imaging and pathology of the cases with hexagonal pattern in HRCT.

This study has several limitations. First, this was a single-center retrospective study, which may be subjected to various biases. External validation studies are warranted in the future. Second, this study included only patients who underwent SLB. Fibrotic HP and IPF, which can be diagnosed by clinical information, HRCT findings, and histological findings by transbronchial forceps biopsies or TBLC, were not included. However, the problem in clinical practice is the differentiation between IPF and fibrotic HP, which requires SLB. It is noteworthy that in this study, the hexagonal pattern was useful for differentiating between the two diseases, even in cases that the diagnosis could not be made based on clinical and imaging findings. Future studies including a cohort comprising of patients with fibrotic HP diagnosed without SLB should be conducted. Finally, we did not examine whether the hexagonal pattern is useful in differentiating fibrotic HP from diffuse lung diseases other than IPF. In the future, we are planning to compare the HRCT findings of fibrotic HP with other diffuse lung diseases.

\section{Conclusion}

Hexagonal pattern is a useful finding for differentiating fibrotic HP from IPF. Further external validation studies should be conducted to evaluate the utility of hexagonal pattern for diagnosing fibrotic HP.

\section{List Of Abbreviations}

HRCT, high-resolution computed tomography

HP, hypersensitivity pneumonitis

IPF, idiopathic pulmonary fibrosis

MDD, multidisciplinary discussion

BAL, bronchoalveolar lavage

TBLC, transbronchial lung cryobiopsy 
SLB, surgical lung biopsy

GGO, ground-glass opacity

UIP, usual interstitial pneumonia

\section{Declarations}

\section{Ethics approval and consent to participate}

This study protocol was reviewed and approved by the Institutional Review Board of Kanagawa Cardiovascular and Respiratory Center, approval number KCRC-20-0023.

Consent to participate statement: Because this study was a retrospective study, written informed consent was not obtained from participants. The opt-out consent was adopted for patient enrollment in this study (approved by the Institutional Review Board of Kanagawa Cardiovascular and Respiratory Center).

\section{Consent for publication}

Not applicable

\section{Availability of data and material}

The dataset supporting the conclusions of this article is presented within the article. The detailed clinical data is not available because of patients' confidentiality.

\section{Competing Interests}

The authors declare that they have no competing interests.

\section{Funding}

This research did not receive any specific grant from funding agencies in the public, commercial, or notfor-profit sectors.

\section{Author contributions}

H. O. takes responsibility for the content of the manuscript, including the integrity of the data and data analysis. D. F. and T. I. was involved in the analysis and interpretation of the HRCT findings. T. T. was involved in the analysis and interpretation of the pathological findings. H. O., T. I., Tsu. O., H. K., T. B., and Ta. O. were involved in the analysis and interpretation of the clinical data. H. O., D. F., T. I., Tsu. O., H. K., T. B., T. T., T. S., and Ta. O. were involved in revising the manuscript. All authors have read and approved the final manuscript.

\section{Acknowledgements}


The authors would like to acknowledge Toshihiro Misumi, PhD who supported the statistical analysis.

\section{Author's information (optinal)}

Not applicable

\section{References}

1. Raghu G, Remy-Jardin M, Ryerson CJ, et al. Diagnosis of hypersensitivity pneumonitis in adults. An official ATS/JRS/ALAT clinical practice guideline. Am J Respir Crit Care Med. 2020;202(3):e36-e69.

2. Fernandez Perez ER, Travis WD, Lynch DA, et al. Diagnosis and evaluation of hypersensitivity pneumonitis: CHEST guideline and expert panel report. Chest. 2021.

3. Lynch DA, Newell JD, Logan PM, King TE, Müller NL. Can CT distinguish hypersensitivity pneumonitis from idiopathic pulmonary fibrosis? AJR Am J Roentgenol. 1995;165(4):807-811.

4. Silva CIS, Müller NL, Lynch DA, et al. Chronic hypersensitivity pneumonitis: differentiation from idiopathic pulmonary fibrosis and nonspecific interstitial pneumonia by using thin-section CT. Radiology. 2008;246(1):288-297.

5. Salisbury ML, Myers JL, Belloli EA, Kazerooni EA, Martinez FJ, Flaherty KR. Diagnosis and treatment of fibrotic hypersensitivity pneumonia. Where we stand and where we need to go. Am J Respir Crit Care Med. 2017;196(6):690-699.

6. Salisbury ML, Gross BH, Chughtai A, et al. Development and validation of a radiological diagnosis model for hypersensitivity pneumonitis. Eur Respir J. 2018;52(2).

7. Barnett J, Molyneaux PL, Rawal B, et al. Variable utility of mosaic attenuation to distinguish fibrotic hypersensitivity pneumonitis from idiopathic pulmonary fibrosis. Eur Respir J. 2019;54(1).

8. Tateishi T, Johkoh T, Sakai F, et al. High-resolution CT features distinguishing usual interstitial pneumonia pattern in chronic hypersensitivity pneumonitis from those with idiopathic pulmonary fibrosis. Jpn J Radiol. 2020;38(6):524-532.

9. Ohtani Y, Saiki S, Kitaichi M, et al. Chronic bird fancier's lung: histopathological and clinical correlation. An application of the 2002 ATS/ERS consensus classification of the idiopathic interstitial pneumonias. Thorax. 2005;60(8):665-671.

10. Lacasse $Y$, Selman $M$, Costabel $U$, et al. Clinical diagnosis of hypersensitivity pneumonitis. Am J Respir Crit Care Med. 2003;168(8):952-958.

11. Vasakova M, Morell F, Walsh S, Leslie K, Raghu G. Hypersensitivity pneumonitis: perspectives in diagnosis and management. Am J Respir Crit Care Med. 2017;196(6):680-689.

12. Raghu G, Remy-Jardin M, Myers JL, et al. Diagnosis of idiopathic pulmonary fibrosis. An official ATS/ERS/JRS/ALAT clinical practice guideline. Am J Respir Crit Care Med. 2018;198(5):e44-e68.

13. Fischer A, Antoniou KM, Brown KK, et al. An official European Respiratory Society/American Thoracic Society research statement: interstitial pneumonia with autoimmune features. Eur Respir J. 2015;46(4):976-987. 
14. Hansell DM, Bankier AA, MacMahon H, McLoud TC, Müller NL, Remy J. Fleischner Society: glossary of terms for thoracic imaging. Radiology. 2008;246(3):697-722.

15. Okada Y. Pulmonary Lymphatic System and Lung Cancer, 1st edn. Kinpodo; 1989.

16. Mitaka K, Miyazaki Y, Yasui M, et al. Th2-biased immune responses are important in a murine model of chronic hypersensitivity pneumonitis. Int Arch Allergy Immunol. 2011;154(3):264-274.

17. Lynch DA, Newell JD, Logan PM, King TE, Jr., Müller NL. Can CT distinguish hypersensitivity pneumonitis from idiopathic pulmonary fibrosis? AJR Am J Roentgenol. 1995;165(4):807-811.

18. Webb WR, Muller NL, Naidich DP. High-Resolution CT of the Lung, 5th edn. Wolters Kluwer; 2014.

19. Giorgia Dalpiaz AC. Atlas of Diffuse Lung Diseases: A Multidisciplinary Approach Springer; 2016.

20. Kumar R, Spalgais S, Ranga V. Hypersensitivity pneumonitis: clinical, radiological and pathological profile of 103 patients from North India. Monaldi Arch Chest Dis. 2020;90(3).

21. Casoni GL, Tomassetti S, Cavazza A, et al. Transbronchial lung cryobiopsy in the diagnosis of fibrotic interstitial lung diseases. PLoS One. 2014;9(2):e86716.

22. Iftikhar IH, Alghothani L, Sardi A, Berkowitz D, Musani Al. Transbronchial lung cryobiopsy and videoassisted thoracoscopic lung biopsy in the diagnosis of diffuse parenchymal lung disease. A metaanalysis of diagnostic test accuracy. Ann Am Thorac Soc. 2017;14(7):1197-1211.

23. Lentz RJ, Argento AC, Colby TV, Rickman OB, Maldonado F. Transbronchial cryobiopsy for diffuse parenchymal lung disease: a state-of-the-art review of procedural techniques, current evidence, and future challenges. J Thorac Dis. 2017;9(7):2186-2203.

24. Hetzel J, Maldonado F, Ravaglia C, et al. Transbronchial cryobiopsies for the diagnosis of diffuse parenchymal lung diseases: expert statement from the cryobiopsy working group on safety and utility and a call for standardization of the procedure. Respiration. 2018;95(3):188-200.

\section{Figures}



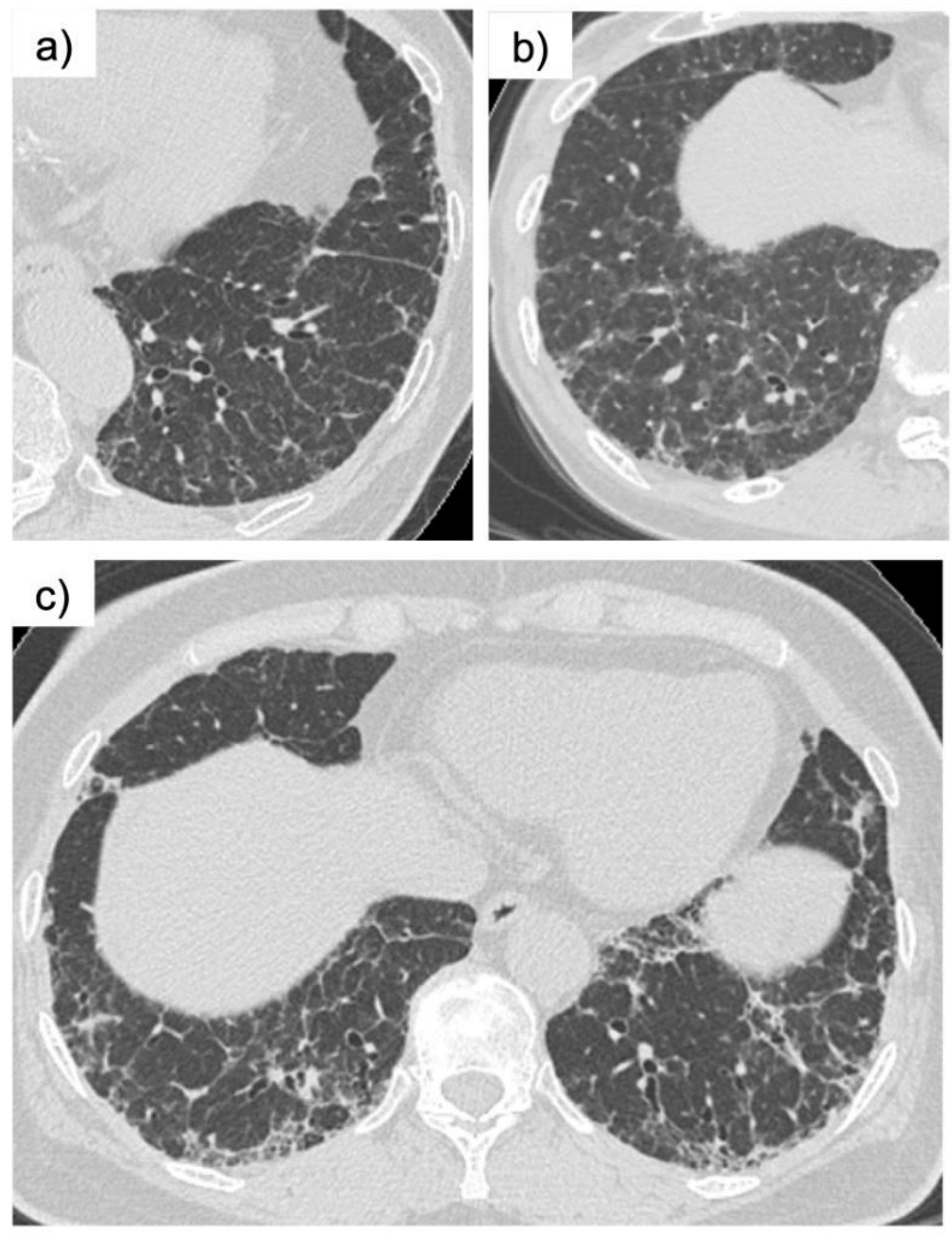

Figure 1

\section{Figure 1}

Hexagonal pattern in the HRCT a, b: Hexagonal pattern is an interlobular septal thickening expanding from the subpleural region to two or more inner layers of the secondary pulmonary lobules $\mathrm{c}$ : When there is subpleural collapse such that the structure of the secondary pulmonary lobules is no longer recognizable, hexagonal pattern was defined as interlobular septal thickening that expands to two or more inner layers of the secondary pulmonary lobules from the collapsed area 

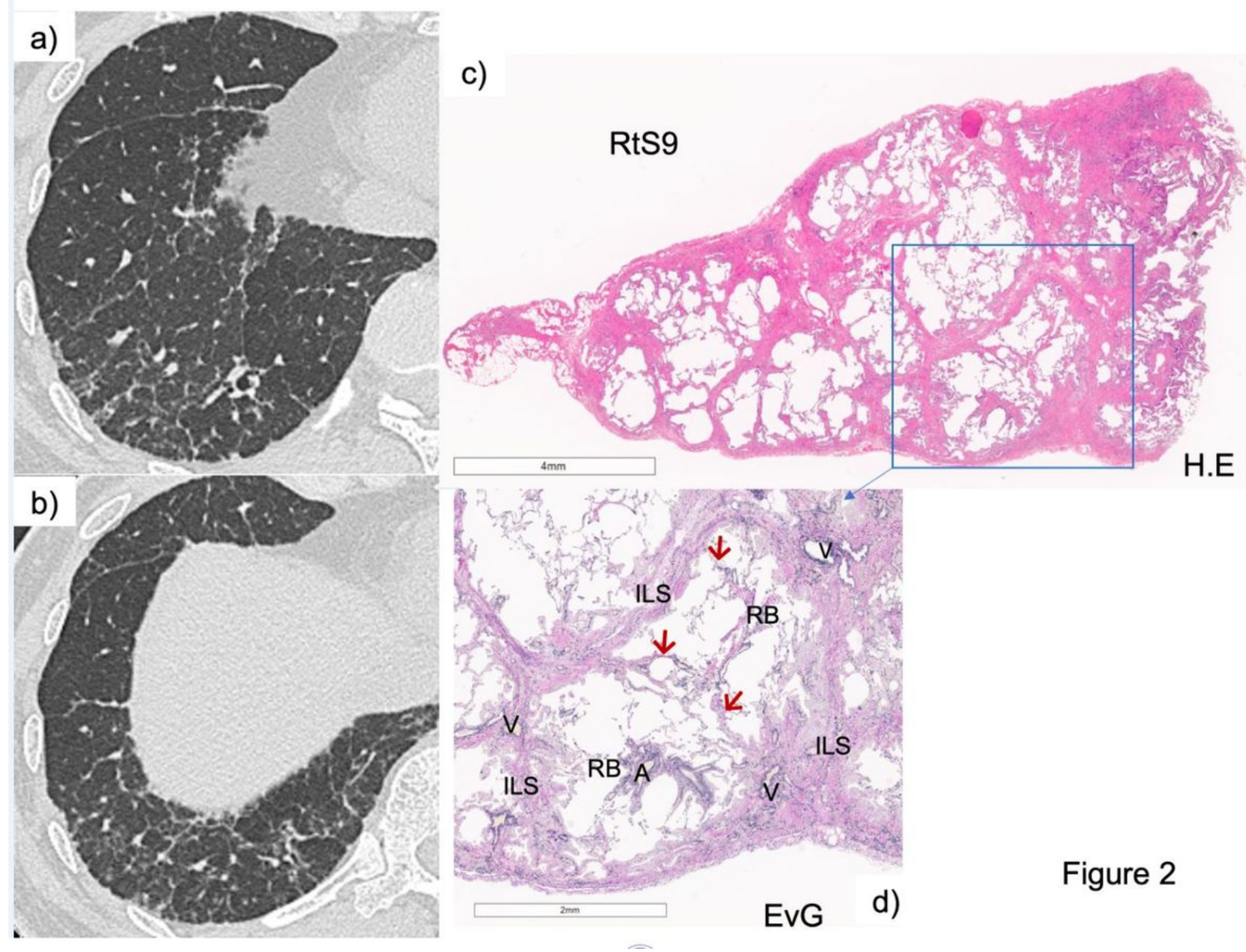

Figure 2

Figure 2

The pathological specimen of the patient with hexagonal pattern in HRCT $a$, b: The HRCT shows interlobular septal thickening expanding from the subpleural region to two or more inner layers of the secondary pulmonary lobules $\mathrm{c}, \mathrm{d}$ : The specimen of right $\mathrm{S} 9$ obtained via surgical lung biopsy. Perilobular fibrosis and bridging fibrosis connecting the fibrosis in the centrilobular area and the fibrosis in the perilobular area were observed. The arrows indicate bridging fibrosis. In this case, the hexagonal pattern observed in the HRCT corresponded to perilobular fibrosis ILS, interlobular septum; V, vein; A, artery; RB, respiratory bronchiole; $\mathrm{HE}$, hematoxylin and eosin; Elastica van Gieson 
Figure 3

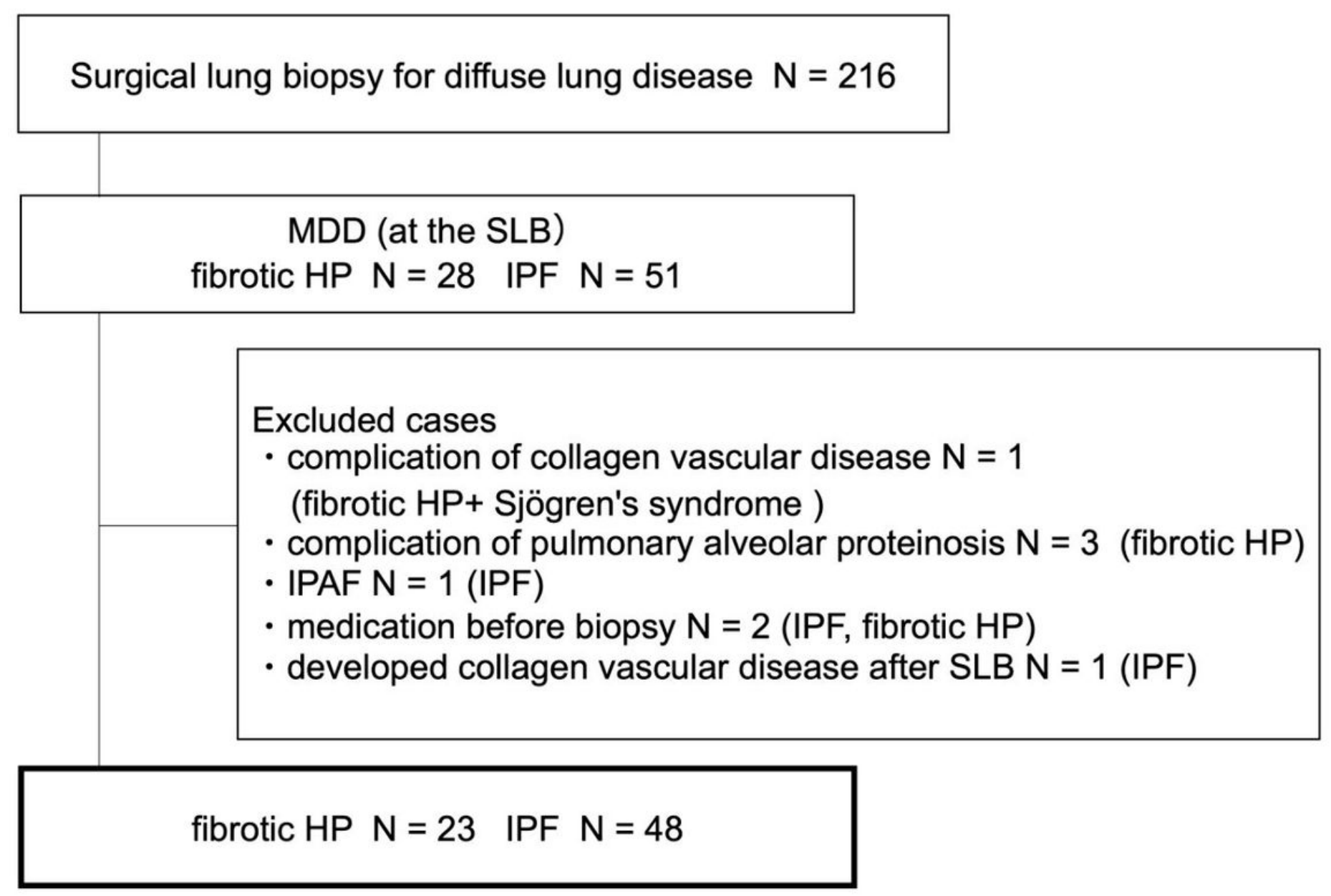

Figure 3

Patient flow diagram MDD, multidisciplinary discussion; SLB, surgical lung biopsy; HP, hypersensitivity pneumonitis; IPF, idiopathic pulmonary fibrosis; IPAF, interstitial pneumonia with autoimmune features 